\title{
Economic Evaluation of Different Organizational Models for the Management of Patients with Hepatitis C
}

\author{
Stefano Fagiuoli ${ }^{1}$, Luisa Pasulo ${ }^{1}$, Franco Maggiolo ${ }^{2}$, Rosaria Spinella ${ }^{3}$, \\ Paolo Del Poggio ${ }^{3}$, Roberto Boldizzoni ${ }^{4}$, Mariella Di Marco ${ }^{5}$, Alessandro Aronica ${ }^{6}$, \\ Chiara Benedetti ${ }^{6}$, Paolo Correale ${ }^{6}$, Chiara Garavaglia ${ }^{6}$, Carlo Nicora ${ }^{7}$ \\ U.O.C. Gastroenterologia - Epatologia e Trapiantologia - ASST Papa Giovanni XXIII, Bergamo \\ U.O.C. Malattie Infettive - ASST Papa Giovanni XXII, Bergamo \\ Medicina Istituti Ospedalieri Bergamaschi, Bergamo \\ 4 U.O.C. Medicina ASST Bergamo Ovest -Treviglio (BG) \\ U.O.C. Medicina A.S.S.T. Bergamo Est - Ospedale "Bolognini" - Seriate (BG) \\ Tefen \& Partners Consulting \\ Direzione Generale - ASST Papa Giovanni XXIII, Bergamo
}

\begin{abstract}
BACKGROUND: Access to Directly Acting Antivirals (DAAs) for Hepatitis C Virus (HCV) treatment in Italy was initially restricted to severe patients. In 2017, AIFA expanded access to all patients, to achieve elimination by 2030.

AIM: To investigate the impact of different hospitals' organizational models on elimination timing, treatment capacity and direct costs.

METHODS: Most Regional healthcare systems in Italy deploy a Center of Excellence (CoE) organizational model, where patients are referred to a single major hospital in the area, which is the only one that can prescribe and deliver DAAs. The study was conducted at Bergamo's (Lombardy, Italy) Papa Giovanni XXIII hospital (PG-23), which deploys a Hub\&Spoke model: the Hub (PG-23) prescribes and delivers DAAs while Spokes (four smaller hospitals) can only prescribe them. The study compares the two models (CoE vs. H\&S). Patient journey and workloads were mapped and quantified through interviews with hospital stakeholders. Cost data were collected through the hospital's IT system; the sample comprised 2,277 HCV patients, over one year.

RESULTS: The study calculated the average cost to treat HCV patients ( $€ 1,470$ per patient). Key cost drivers are lab tests $(60 \%)$ and specialist visits $(30 \%)$. Over one year, H\&S can treat $68 \%$ more patients than CoE. As deferred patients absorb up to $40 \%$ of total costs, the "Optimized" model was designed by streamlining specialists' visits and involving general practitioners during follow-up. "Optimized" model increases treatment capacity and reduces costs of deferred patients by $72 \%$ vs $\mathrm{CoE}$.

CONCLUSION: The study demonstrates the importance of organizational models in efficiently achieving 2030 elimination.
\end{abstract}

\section{Keywords}

Hepatitis C; Italy; Models, Organizational; Antiviral Agents; DAA; HCV; Hub \& Spoke; Center of Excellence

\section{BACKGROUND}

Hepatitis $\mathrm{C}$ is an infective liver disease affecting globally more than 71 million people and causing 399,000 deaths each year [1]. The disease burden encouraged the World Health Organization (WHO) to launch a program aiming at eliminating Hepatitis C Virus (HCV) by 2030 [2].

Although 15-45\% [1] of infected people can recover within 6 months of infection without any treatment, the chronic infection caused by HCV often leads to the development of cirrhosis, hepatocellular carcinoma (HCC) and several other comorbidities that complicate patient conditions.

In Italy the Italian Medicines Agency (AIFA) estimates that there are up to 300,000 infected $\mathrm{HCV}$ patients $(0.5 \%$ prevalence). The mortality rate of $\mathrm{HCV}$-related diseases still accounts for 10,000 cases a year [3].

Corresponding author Dr. Stefano Fagiuoli sfagiuoli@asst-pg23.it

Received: 26 September 2018 Accepted: 11 December 2018 Published: 28 January 2019 
- A multidisciplinary team made up by a liver disease expert (gastroenterology, infectious disease, general medicine), a pathologist and a dedicated nurse. All team members must be skilled in viral hepatitis diseases

- A defined team coordinator

- A nurse skilled in patient education and treatment

management

- Current management of at least 500 hepatic patients

- A radiology unit

- A digestive endoscopy unit

- A serology laboratory

- A day hospital service for hepatic patients

- A pharmacy to dispense DAA, provide counseling in

compliance with AIFA requirements

-An integrated path with the regional transplant network
- An access to a multidisciplinary day hospital to diagnose and

treat hepatic patients

- Clinical research activities

- A virology laboratory

- A laboratory specialized in drugs serum level monitoring

Table I. Key organizational characteristics that a HCV Centre of Excellence must have/ensure

For a long time, HCV has been difficult to treat and the only available pharmacological therapies, interferon and ribavirin, did not ensure full patient recovery nor absence of side-effects. Starting in 2011, Directly Acting Antivirals (DAAs) have been introduced in Italy, representing an initial step towards virus elimination. The first generation of DAAs (telaprevir and boceprevir) reached a $75 \%$ of sustained virologic response (SVR) in clinical trials [4,5]. Second generation of DAAs and their combinations (sofosbuvir, simeprevir, daclatasvir, ledipasvir, ombitasvir/ paritaprevir/ritonavir, dasabuvir, elbasvir/grazoprevir) significantly increased SVR up to $95 \%$ and showed rare side-effects [6]. The wave of third generation of DAAs (sofosbuvir/velpatasvir, glecaprevir/pibrentasvir), introduced in 2016/2017, represented a further improvement in treatment: new pangenotypic drugs treating patients with a SVR $>97.5 \%$ in less than 12 weeks [6].

However, access to DAAs in Italy was initially limited by AIFA to patients presenting the most severe levels of the disease and with life-threatening conditions (i.e., fibrosis higher than 3 or patients with critical extra-hepatic complications). On top of that, only a selected number of hospitals, qualifying as Centre of Excellence $(\mathrm{CoE})$, were allowed to prescribe and distribute DAAs [7]. The decisions to limit both access criteria and prescribing centers were taken to ensure treatment economic sustainability, considering the high HCV prevalence across the Country and the substantial impact of DAA costs on Italian National Healthcare System expenditure [8].

Following the introduction of DAAs, Lombardy's Healthcare System (one of the 21 different Regional Healthcare Systems in Italy) developed a new integrated care pathway [9] for $\mathrm{HCV}$, explicitly calling for key organizational requirements a hospital would need to meet in order to be qualified as a $\mathrm{CoE}$ (refer to Table I).

ASST-Papa Giovanni XXIII (PG-23) qualified as CoE and started dispensing DAAs to $\mathrm{HCV}$ patients in the Bergamo area (Lombardy, Italy), commencing from mid-2015. During this period, in compliance with regional regulation and aiming to increase treatment capacity as well as to ensure treatment continuity for patients, the PG-23 designed and implemented a new Hub\&Spoke organizational model comprising the PG-23 itself, as the Hub and the only hospital in the area authorized as a DAA prescriber and distributor, and other six smaller hospitals as Spokes. The introduction of the Spokes not only allowed to increase the number of access points, but also offered the opportunity for existing patients to be referred to the center where they had received previous treatments (e.g. interferon and ribavirin) and for new ones to be directly referred to the closest hospital. Notwithstanding the efforts of healthcare providers to improve treatment access, and as a consequence of AIFA's access policy, a relevant number of HCV affected patients could not be treated with DAAs in Italy. Finally, as of mid2017, AIFA expanded access to DAAs for all HCV patients [10]; the rationale behind such decision was achieving disease elimination in 2030 , by treating about 80,000 patients per year.

Considering that extending HCV treatment to patients in any fibrosis stage has been proved to improve health outcomes and is cost-effective [11], is now pivotal to understand what is the organization model able to accelerate access to treatment as well as ensuring its economic sustainability.

The study aim was therefore threefold:

1. Investigate the Hub\&Spoke organizational model deployed by PG-23 and assess its performance in terms of $\mathrm{HCV}$ patient treatment capacity and direct hospital costs. 
2. Identify areas of improvement in the organizational model currently deployed by PG23 and design an "optimized" organizational model improving the performance on the Hub\&Spoke one.

3. Compare three different organizational models (CoE, Hub\&Spoke, "optimized") to understand their impact on elimination timing and cost effectiveness, with regards to AIFA's new access criteria.

\section{METHODS}

\section{Study Design}

Study design consists of four main steps. Firstly, patient journey was mapped. We employed a swim lane chart approach to capture in detail key activities and interactions among the involved hospital stakeholders in HCV DAA patient journey.

Secondly, we quantified workloads for each activity of the journey performed by hospital stakeholders within PG-23 Hub\&Spoke organization. Workload data were collected through 24 interviews with hospital stakeholders involved in the patient journey ( $48 \%$ of total), including:

- Specialists (hepatologists and infectious diseases specialists);

- Nurses;

- Psychologists/psychiatrists;

- Pharmacists and pharmacy assistants;

- Laboratory technicians and directors.

Through interviews, we identified key improvement opportunities in the current organizational model. Improvement opportunities were used to design a new "optimized" model, aiming to improve patient's access to treatment.

Thirdly, we collected all relevant data input needed to quantify patient journey direct costs. Cost data were collected through PG-23 data owners (e.g. Controlling Department) while we leverage on an existing Lombardy Regional database to collect historical patients related information (e.g. DAA treatment period, genotype, fibrosis).

Finally, a parametric model was developed to assess the impact of the three organization models (CoE, Hub\&Spoke, "optimized") on elimination timing, treatment capacity and direct costs.

\section{Study Population}

Data sample included patients treated over one year (June $30^{\text {th }} 2015$ - June $30^{\text {th }} 2016$ ) in any of the seven hospitals part of PG-23's Hub\&Spoke organization:

1. ASST Ospedale Papa Giovanni XXIII (PG-23);

2. ASST-Bergamo Est (Bolognini, Seriate);

3. ASST-Bergamo Est (M.O.A. Locatelli, Piario);

4. ASST-Bergamo Ovest (A.O. S. Giovanni Bianco, Treviglio);

5. ASST-Bergamo Ovest (Ospedale "Treviglio-Caravaggio", Treviglio);

6. Policlinico Ponte San Pietro;

7. Policlinico San Marco.

All patients were either HCV mono-infected, or HBV co-infected. The HBV coinfection had no impact on the HCV patient journey. Only patients treated with DAAs were included in study population (> $98 \%$ of all HCV patients). Data sample was extracted from a Lombardy Health Service database.

Patient data included clinical and general information such as genotype, fibrosis, hospital name, type and length of therapy, presence of co-morbidities.

We collected specific comorbidities data for HCV patients treated by the gastroenterology unit of PG-23 (321 patients out of 579 patients treated in total by all five hospitals). The comorbidity distribution of the sub-sample was then applied to the wider study population.

\section{New AIFA Access Criteria}

All patients in the study population were eligible to DAA treatment according to AIFA access criteria in place until March 31 2017.

From March 31 $1^{\text {st }}$, 2017, AIFA released 11 new access criteria that extended the access of treatment to all $\mathrm{HCV}$ patients, regardless of fibrosis level or other clinical parameters [12]. New access criteria were used in the parametric model to simulate the expansion of patient population eligible for DAA treatments. 


\section{Deferred and Stand-by Patient Sample}

On top of treated patients (i.e., patients who received DAA treatment in the study timeframe), this study analyzed two additional groups of patients:

1. Deferred patients: patients who met AIFA access criteria in place before March $31^{\text {st }}, 2017$ but could not receive treatment immediately, and were added to the waiting list;

2. Stand-by patients: patients who did not meet AIFA access criteria in place before March $31^{\text {st }}, 2017$; F0-F1-F2 patients represent most of this group.

The number of deferred patients was calculated using Bergamo Hub\&Spoke's actual waiting list as of January 2017. The number of stand-by patients was estimated through interviews with PG-23's specialists and was based on their experience in the diagnosis phase of the patient journey.

We did not consider currently undiagnosed patients in the study, as we did not explore any relationship between them and the hospital's organizational model.

\section{Direct Hospital Costs}

Direct hospital costs were calculated using four types of data:

1. Internal cost (in $€$ ) per hour of each stakeholder involved in the HCV patient journey;

2. Internal cost (in $€$ ) of each microbiology and virology test, including lab equipment;

3. Internal cost (in $€$ ) of Fibroscan testing;

4. Workload (in minutes) for each hospital stakeholder and each activity of the HCV patient journey.

Analysis did not include general\&administative hospital costs, additional costs for patients resistant to DAA treatment ( $<5 \%$ of patient population) and cost of HCV reinfection. Direct hospital costs also did not include the cost of purchasing DAA drugs, which are fully reimbursed by the Healthcare System.

Laboratory costs included cost per kit, supplementary disposables and equipment amortization.

\section{Organizational Models}

The study analyzed three different organizational models: CoE, Hub\&Spoke and "optimized" model.

\section{Center of Excellence (CoE)}

$\mathrm{CoE}$ is the model originally designed by Lombardy Health Care system, where patients are referred to a single major hospital in the area, the only one authorized to prescribe and distribute DAAs (Table I). Patients can access the CoE through multiple access points (e.g. Gastroenterology units, Infectious disease units, General medicine, etc.) in the area.

Although the model ensures the delivery of standardized diagnostic/therapeutic flow in the area, it can limit continuity of treatment. Indeed, patients who were already enrolled for a previous HCV treatment (i.e., with interferon and ribavirin) in a different hospital than the CoE need to move to the CoE to receive DAA treatment, thus generally increasing logistical costs and time spent by patients and caregivers. Moreover, specialists and other stakeholders employed at non-CoE hospitals in the area are not adding to HCV treatment capacity. CoE analysis in the parametric model was performed assuming PG-23 as a standalone center.

\section{Hub\&Spoke}

Hub\&Spoke is the model implemented by PG-23 and Bergamo-area hospitals, where the Hub (PG-23) is the only hospital in the network authorized as a DAA prescriber and distributor. The other four smaller hospitals deploy specialists, formally employed by the Hub, who can prescribe DAA treatments.

The model involves a total of 48 clinical stakeholders in HCV care: 24 specialists, 6 nurses, 1 psychologist, 1 psychiatrist, 3 pharmacists, 1 pharmacist assistant, 2 laboratory directors and 10 laboratory technicians.

Hub\&Spoke provides patients with widespread accessibility, ensuring treatment continuity. The continuity is applied to all patients, including those previously enrolled in Spoke centers (e.g. patients treated with interferon and ribavirin). Each center is able to prescribe DAAs; however, supply and dispensing are managed centrally by the Hub.

Four nurses, available in three out of the five hospitals of the Hub\&Spoke network, were dedicated to the HCV treatment. Nurses support specialists for patients scheduling, document preparation, medical services dispensing and patient's condition monitoring. 


\section{"Optimized"}

Three optimization opportunities were identified and selected to design the "optimized" model:

1. Involvement of GPs to follow-up on patients without comorbidities. It was observed that the effectiveness/SVR of DAAs can allow specialists to discharge patients without comorbidities/side-effects once treatment is completed. Hence, General Practitioners (GPs) could handle the follow-up of these selected patients, leveraging also on telemedicine systems enabling GPs to remotely upload and share analyses with specialists as well as to ask for second opinions. The opportunity can thus decrease specialist's workload by having three follow-up visits per patient executed by GPs.

2. Reduction of in-treatment monitoring visits. Considering both significant SVR and safety observed for DAAs treatment as well as the expansion of access criteria, we identified the opportunity to skip standard monitoring visits, without generating any additional risks for the patient. Therefore, the specialist in charge of a given patient can decide whether to schedule a patient visit or not on a visit-by-visit basis, depending on his/her assessment of monthly laboratory tests results. The opportunity can thus decrease specialist's workload; we estimate that a minimum of one and a maximum of five standard monitoring visits per patient will not be executed.

3. Streamlining of diagnosis visits. We noticed that a statistically significant number of patients was receiving two visits in the diagnosis phase before starting treatment; part of this effort was related to specialists needing to differentiate between patients meeting/not meeting AIFA access criteria (in place before March 2017). Thanks to new access criteria, the patient journey can be simplified by having a single visit including the second diagnosis visit and the first treatment visit.

Three additional improvement opportunities were identified, but not included in the "optimized" model; we indicate them below as a reference for other healthcare providers:

- GPs can support specialists by performing follow-up of patients affected by mild comorbidities. The GP would be always able to reach specialist through telemedicine systems to share patient's clinical data and require a second opinion;

- Drug distribution for DAAs is currently centralized in the Hub structure: a new process was designed to improve drug distribution from the Hub to each Spoke, in compliance with AIFA guidelines;

- Create a task force ("Hepatitis Centre") involving stakeholders from all relevant Departments (i.e. Gastroenterology, Infectious Disease, General Medicine) to improve efficiency and accuracy in disease diagnosis.

\section{Parametric Model Design}

A parametric model was designed to compare the three different organizational models and assess their impact on disease elimination timing, treatment capacity and direct costs. Using current treatment capacity and direct cost data collected, the parametric model simulated the performance of each organizational model starting from June $30^{\text {th }}$, 2016 to waiting list depletion.

We calculated waiting lists with month granularity, by dividing the number of untreated patients as of June $30^{\text {th }}, 2016$ by each organizational model's monthly treatment capacity. Notably, if AIFA access criteria in place before April 1 1 ${ }^{\text {st }}, 2017$ are considered, untreated patients correspond to previously defined as "deferred" patients; if new AIFA access criteria are considered, untreated patients correspond to the sum of "deferred" patients and previously defined as "stand-by" patients. Newly diagnosed patients were added to the number of untreated patients until waiting list depletion, assuming a fixed annual increase of $5 \%$.

Waiting list depletion was defined as the point in time (with month granularity) when the number of untreated patients would be equal to or below zero. Waiting list depletion was used as a proxy of disease elimination's timing in the hospital's area. Therefore, an organizational model with a higher treatment capacity would display a smaller waiting list, as well as a shorter time to waiting list depletion and disease elimination.

The parametric model was firstly used to compare CoE and Hub\&Spoke considering access criteria in place until March $30^{\text {th }}$, 2017. Secondly, the parametric model was used to compare CoE and Hub\&Spoke considering new AIFA access criteria. The objective of these two analyses was to identify the most effective and efficient model between CoE and Hub\&Spoke, considering two alternative access scenarios. Finally, we used the parametric model to compare the Hub\&Spoke model with the "optimized" one, considering new AIFA access criteria and with the objective of assessing improvement opportunities embedded in the "optimized" model. 


\section{RESULTS}

\section{HCV Patient Journey}

Three main areas were mapped: diagnosis, treatment and follow-up.

Patients have access to the hospital through three different channels: (i) referral of GPs or other hospital, (ii) currently inpatient moving to outpatient care in the same hospital, (iii) non-treated patient subject to follow-up in the same hospital.

Once a patient accesses the hospital, a first visit with the specialist is organized. The specialist verifies previous diagnosis, if any, recent laboratory analyses and prescribes additional lab tests. Finally, the specialist assesses the severity of the disease to decide whether to start the treatment or defer the patient.

Treatment length, mostly varying between 12-24 weeks, is linked to the selected therapeutic scheme. Every four weeks, patient repeats lab tests, undergoes a visit with the specialist and collects new 28-days DAA medication.

Once treatment is completed, patient receives a follow-up program developed according to patient's condition and the presence of any comorbidities; a standard follow-up includes three visits with the specialist 4, 12 and 24 weeks after treatment conclusion and a final fibrosis assessment with a Fibroscan 12 weeks after treatment conclusion.

\section{Patient Sample}

Final patient sample included a total of 2,277 patients: 579 treated patients, 467 deferred patients and 1,231 stand-by patients.

\begin{tabular}{lcc|ccc|cccc}
\hline & \multicolumn{2}{c}{ Diagnosis } & \multicolumn{2}{c|}{ Treatment } & \multicolumn{2}{c}{ Follow-up } & \multicolumn{2}{c}{ Total } \\
\cline { 2 - 9 } & $\boldsymbol{\epsilon}$ & Hours & $\boldsymbol{\epsilon}$ & Hours & $\boldsymbol{\epsilon}$ & Hours & $\boldsymbol{\epsilon}$ & Hours \\
\hline Specialist & 105 & 1.7 & 249 & 4.1 & 119 & 2 & 473 & 7.8 \\
Nurse & 17 & 0.6 & 38 & 1.3 & 20 & 0.7 & 75 & 2.6 \\
Fibroscan & 17 & - & - & - & 17 & - & 34 & - \\
Lab technician & 23 & 0.9 & 49 & 1.9 & 38 & 1.4 & 110 & 4.2 \\
Lab Director & 8 & 0.3 & 53 & 0.9 & 41 & 0.7 & 102 & 1.9 \\
Lab Test & 185 & - & 255 & - & 196 & - & 636 & - \\
Pharmacist & - & - & 25 & 0.4 & - & - & 25 & 0.4 \\
Pharmacist Assistant & - & - & 15 & 0.5 & - & - & 15 & 0.5 \\
Psychiatrist/Phycologist & 2 & 0.1 & 6 & 0.1 & - & - & 8 & 0.2 \\
Total & 357 & 3.5 & 690 & 9.2 & 431 & 4.8 & & 8 \\
\hline
\end{tabular}

Table II. Average direct costs for one patient treated in Hub\&Spoke model

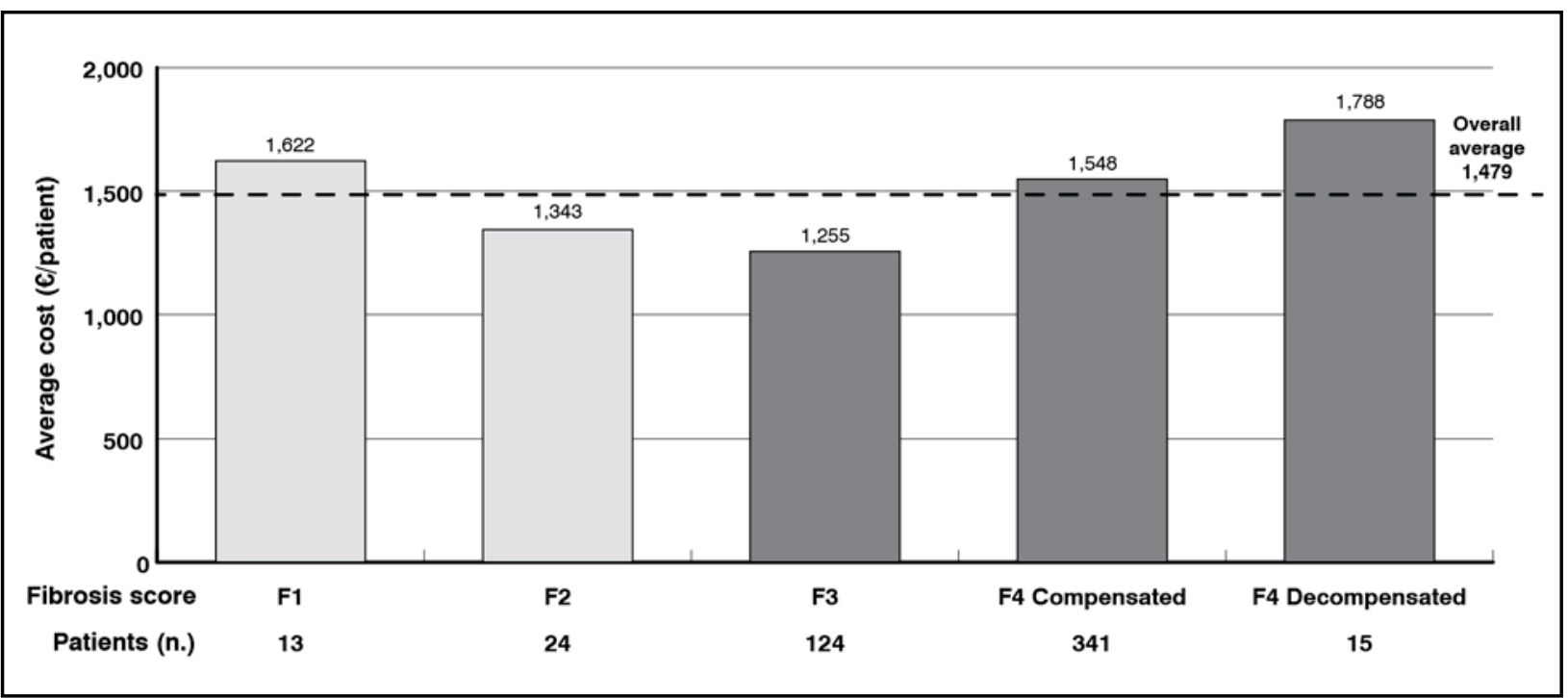

Figure 1. Average direct costs per patient, by fibrosis score 
We observed the presence of four main non-infective comorbidities related to HCV: diabetes $(20 \%)$, ischemic cardiopathy $(7 \%)$, cryoglobulinemia $(6 \%)$ and hepatic insufficiency (3\%). Co-infected HBV patients account for $17 \%$ of total study population.

If new access criteria were applied to study population, deferred patients would increase to $1,698(+263 \%)$ as all stand-by patients would become part of the deferred patients group.

\section{Direct hospital costs}

Total direct hospital costs are $€ 1.4 \mathrm{M}$ at PG-23's Hub\&Spoke model.

Direct costs for treated patients at PG-23's Hub\&Spoke model are equal to $€ 843,735$, average cost for patient is $€ 1,479$ (Table II). Specialists costs ( $€ 473 /$ patient) and lab tests $(€ 636 /$ patient) represent key cost element of the patient journey. Other relevant direct costs are represented by lab technicians and director $(€ 110$ /patient and $€ 102 /$ patient, respectively), nurses (€ 76/patient), Fibroscan test (€ 34/patient), pharmacists and pharmacy assistants ( $€ 25 /$ patient and $€ 15 /$ patient, respectively) and psychologists/psychiatrists ( $€ 8 /$ patient).

F3 and F4 patients represented 93\% of sample; F4 patients were mostly treated in 24 weeks whereas F3 patients in 12 weeks. 7\% of the sample was composed by F1-F2 patients treated with DAAs due to their exceptional clinical record: they either received a liver transplant or were affected by severe extrahepatic diseases. Decompensated cirrhotic patients incurred the highest direct hospital costs ( $€ 1,788$ vs. average cost of $€ 1,479)$ (Figure 1$)$.

Genotype 1 was reported in $66 \%$ of patients, of which $12 \%$ was represented by patients with genotype $1 \mathrm{a}$ and $54 \%$ by patients with genotype $1 \mathrm{~b}$. Genotype $3(\approx 10 \%$ of treated patients) generated the highest cost per patient $(€ 1,730$ versus average cost of $€ 1,479)$.

Both deferred and stand-by patients produced a yearly cost of $€ 351(\approx 25 \%$ of a treated patient), of which more than $60 \%$ is for virologic and microbiologic laboratory tests. Overall, deferred and stand-by patients accounted for $41 \%(\approx € 597,000)$ of total direct hospital costs. In particular, deferred patients accounted for $28 \%$ of such costs $(\approx € 165,000)$, whereas standby patients represented $72 \%(\approx € 432,000)$.

Average direct costs for one patient treated in "optimized" model are reported in Table III.

\section{Parametric Model Results}

We firstly compared $\mathrm{CoE}$ and Hub\&Spoke considering AIFA limited access criteria effective until March $30^{\text {th }}, 2017$. Hub\&Spoke is capable of treating a higher number $(\approx+70 \%)$ of patients over 12 months (579 vs. 343). Waiting list is consequently shorter for Hub\&Spoke: 10 months for Hub\&Spoke compared to 27 months for CoE. In terms of direct costs, cost per treated patient does not significantly differ between the two models (albeit it would be possible to infer that costs sustained by patients and caregivers are lower in Hub\&Spoke, thanks to hospital proximity). We also observed a correlation between treatment capacity and cost efficiency: in fact, while over one year Hub\&Spoke reported higher costs for treated patients $(\approx+70 \%)$ due to the larger number of treated patients, it displayed lower costs for deferred patients $(€-83,000)$ thanks to a shorter waiting list. Cost savings of lowering the waiting list clearly increase over time in the Hub\&Spoke model: considering the same number of patients will be eventually treated by both models, total direct costs down to waiting list depletion

\begin{tabular}{|c|c|c|c|c|c|c|c|c|}
\hline & \multicolumn{2}{|c|}{ Diagnosis } & \multicolumn{2}{|c|}{ Treatment } & \multicolumn{2}{|c|}{ Follow-up } & \multicolumn{2}{|c|}{ Total } \\
\hline & $€$ & Hours & $€$ & Hours & $€$ & Hours & $€$ & Hours \\
\hline Specialist & 84 & 1.4 & 225 & 3.6 & 65 & 0.9 & 374 & 5.9 \\
\hline Nurse & 15 & 0.5 & 35 & 1.2 & 8 & 0.3 & 58 & 2 \\
\hline Fibroscan & 17 & - & - & - & 17 & - & 34 & - \\
\hline Lab technician & 23 & 0.9 & 49 & 1.9 & 38 & 1.4 & 110 & 4.2 \\
\hline Lab Director & 8 & 0.3 & 53 & 0.9 & 41 & 0.7 & 102 & 1.9 \\
\hline Lab Test & 185 & - & 255 & - & 196 & - & 636 & - \\
\hline Pharmacist & - & - & 25 & 0.4 & - & - & 25 & 0.4 \\
\hline Pharmacist Assistant & - & - & 15 & 0.5 & - & - & 15 & 0.5 \\
\hline Psychiatrist/Phycologist & 2 & 0.1 & 6 & 0.1 & - & - & 8 & 0.2 \\
\hline Total & 335 & 3 & 664 & 9 & 366 & 3 & & \\
\hline
\end{tabular}

Table III. Average direct costs for one patient treated in "optimized" model 
equal to $€ 3.82 \mathrm{M}$ for the $\mathrm{CoE}$ and $€ 3.58 \mathrm{M}$ for the Hub\&Spoke model, with the latter generating a saving of $€ 246,813$ (Figure 2).

We secondly compared CoE and Hub\&Spoke considering new AIFA access criteria. Compared to the first scenario, treatment capacity remains constant in both models while waiting lists substantially increase due to 1,231 stand-by patients gaining access to DAA treatments. Waiting list for Hub\&Spoke in this scenario is equal to 4.5 years, compared to 8.7 years for CoE. Furthermore, waiting list depletion corresponds to disease elimination as all patients are eligible for DAA treatment. Focusing once more on total direct costs (including both treated and deferred patients) down to waiting list depletion, CoE costs exceed $€ 8 \mathrm{M}$ over nine years while Hub\&Spoke costs are equal to $€ 6.2 \mathrm{M}$ over the five years needed to deplete the waiting list (Figure 3).

Finally, we compared "optimized" and Hub\&Spoke considering new AIFA access criteria. We observed that the implementation of optimization opportunities generated additional benefits firstly in terms of treatment capacity. In fact, specialist workload would decrease by $15 \%$ and annual access capacity would increase by $28 \%$ (755 patients vs. 579 of Hub\&Spoke). Thus the "optimized" model would deplete the waiting list in 2.9 years only, roughly 1.6 year $(-35 \%)$ less than Hub\&Spoke. Once again, a shorter waiting list leads to cost savings: "op-

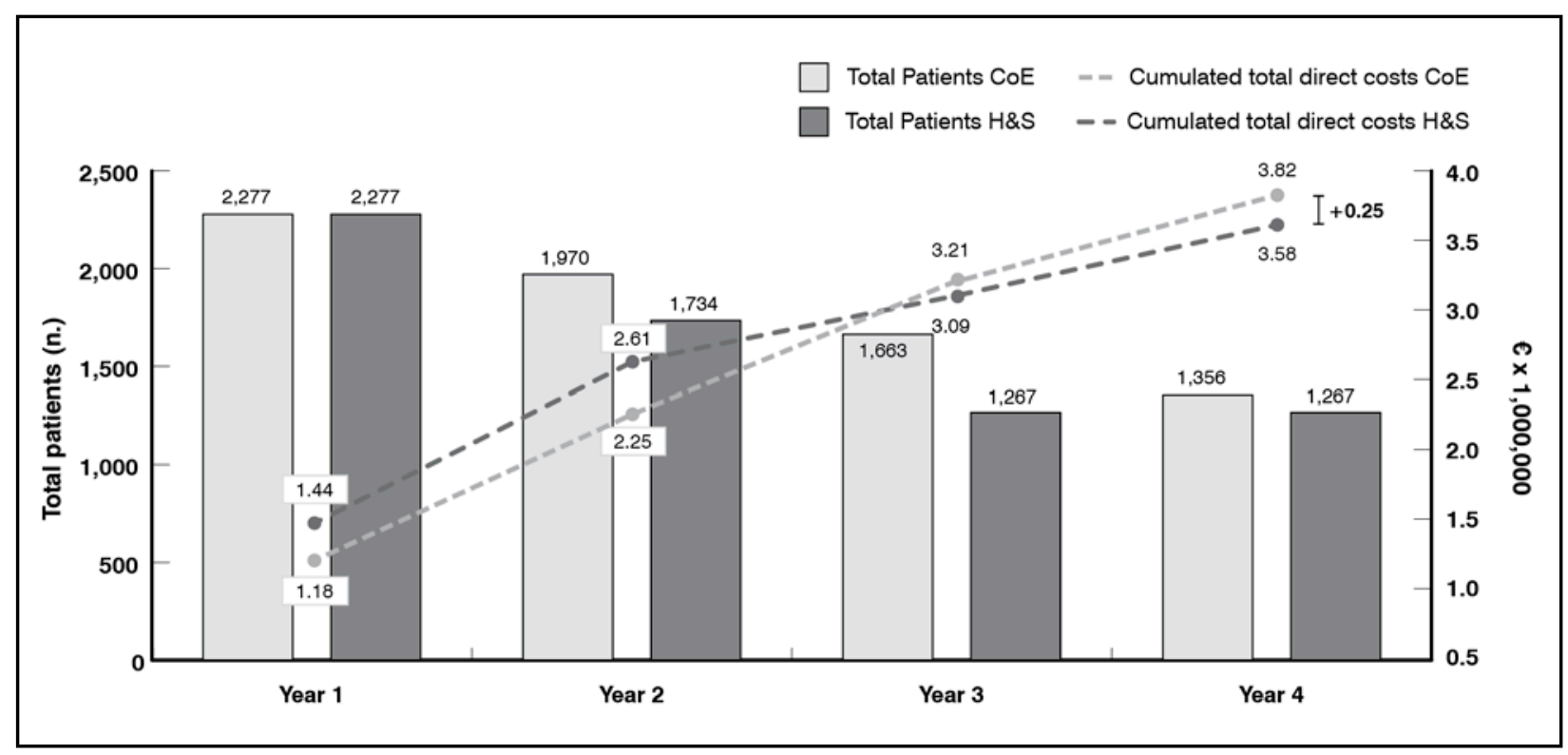

Figure 2. CoE vs. Hub\&Spoke Model in AIFA limited access criteria scenario

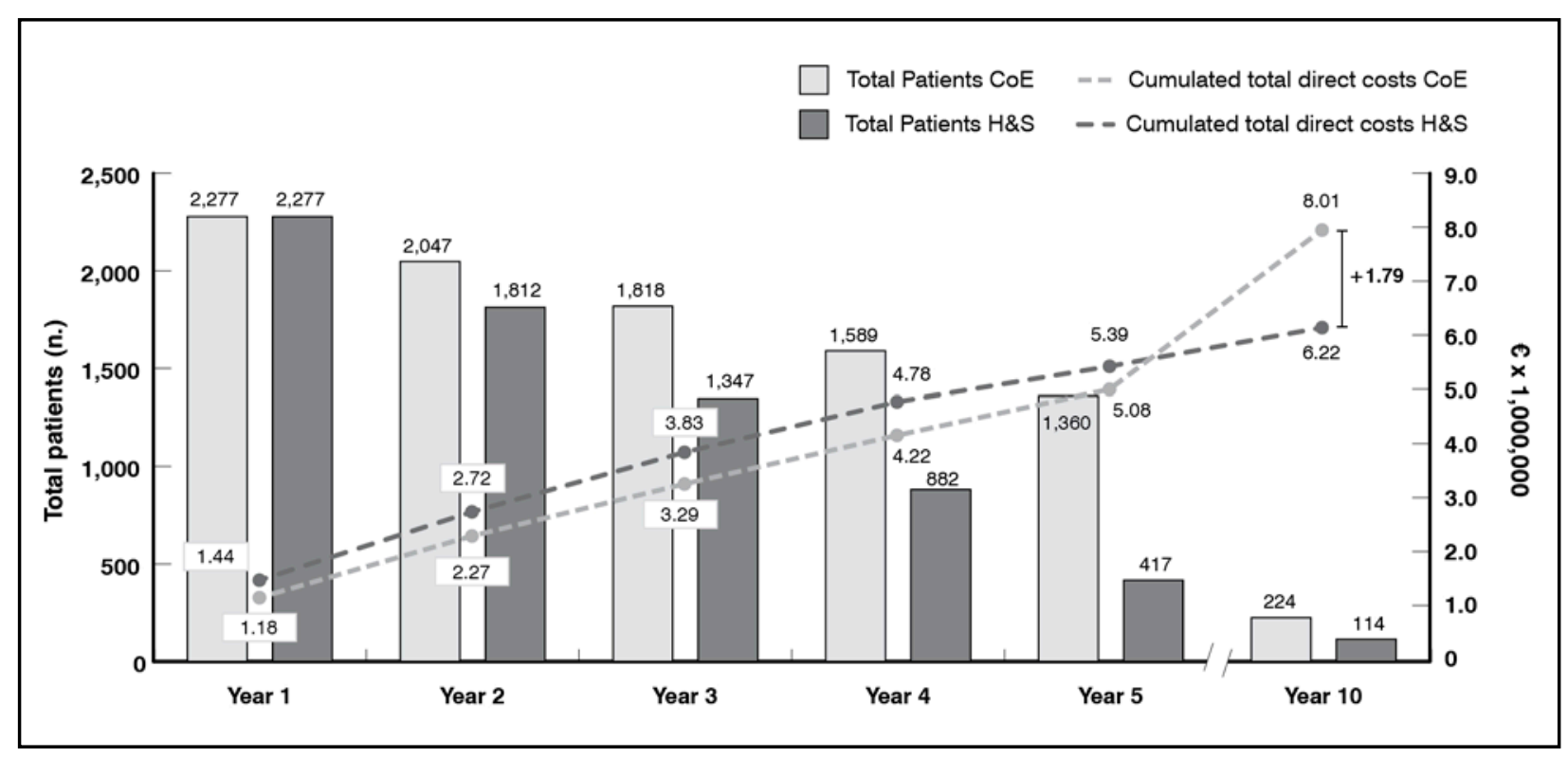

Figure 3. CoE vs. Hub\&Spoke Model in new AIFA 2017 criteria scenario 


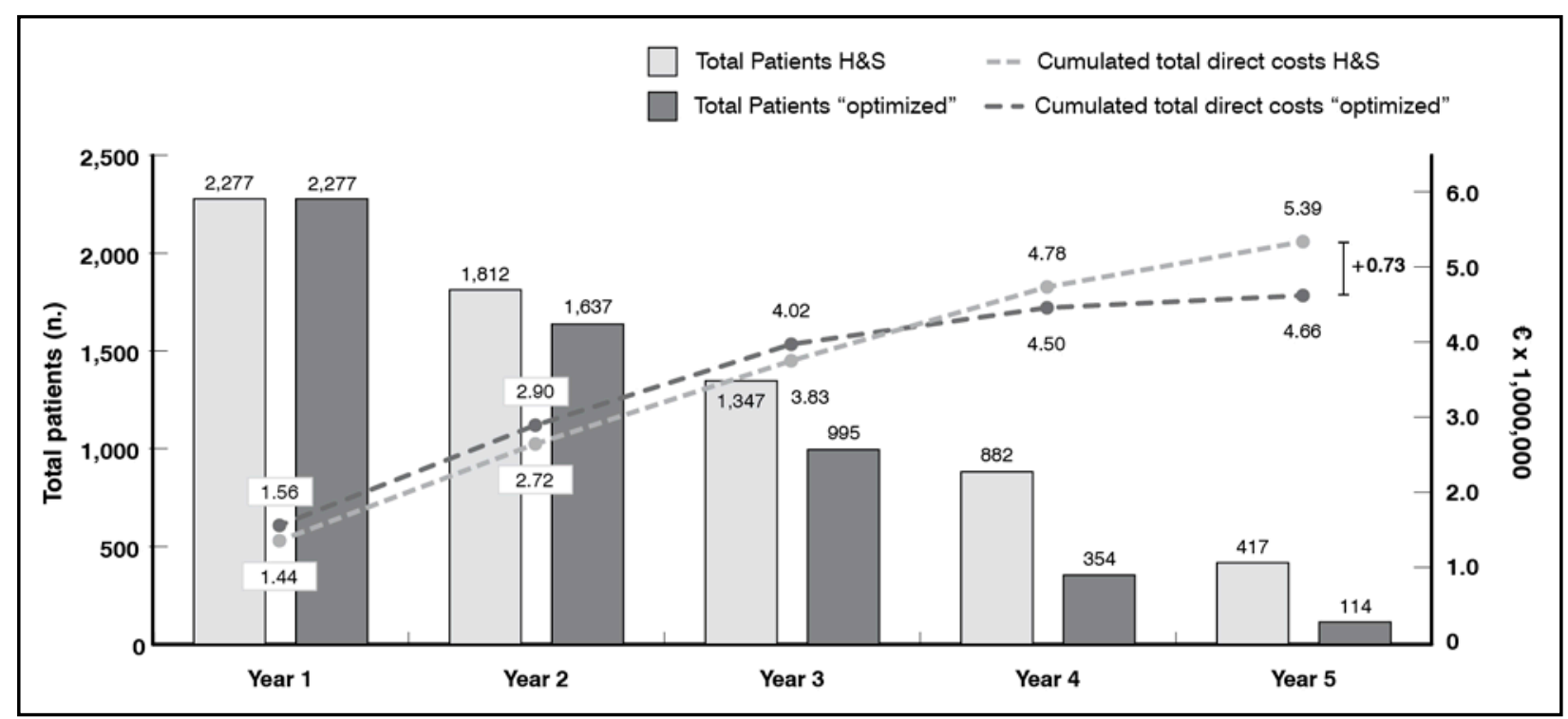

Figure 4. "Optimized" model vs. Hub\&Spoke model in new AIFA 2017 criteria scenario

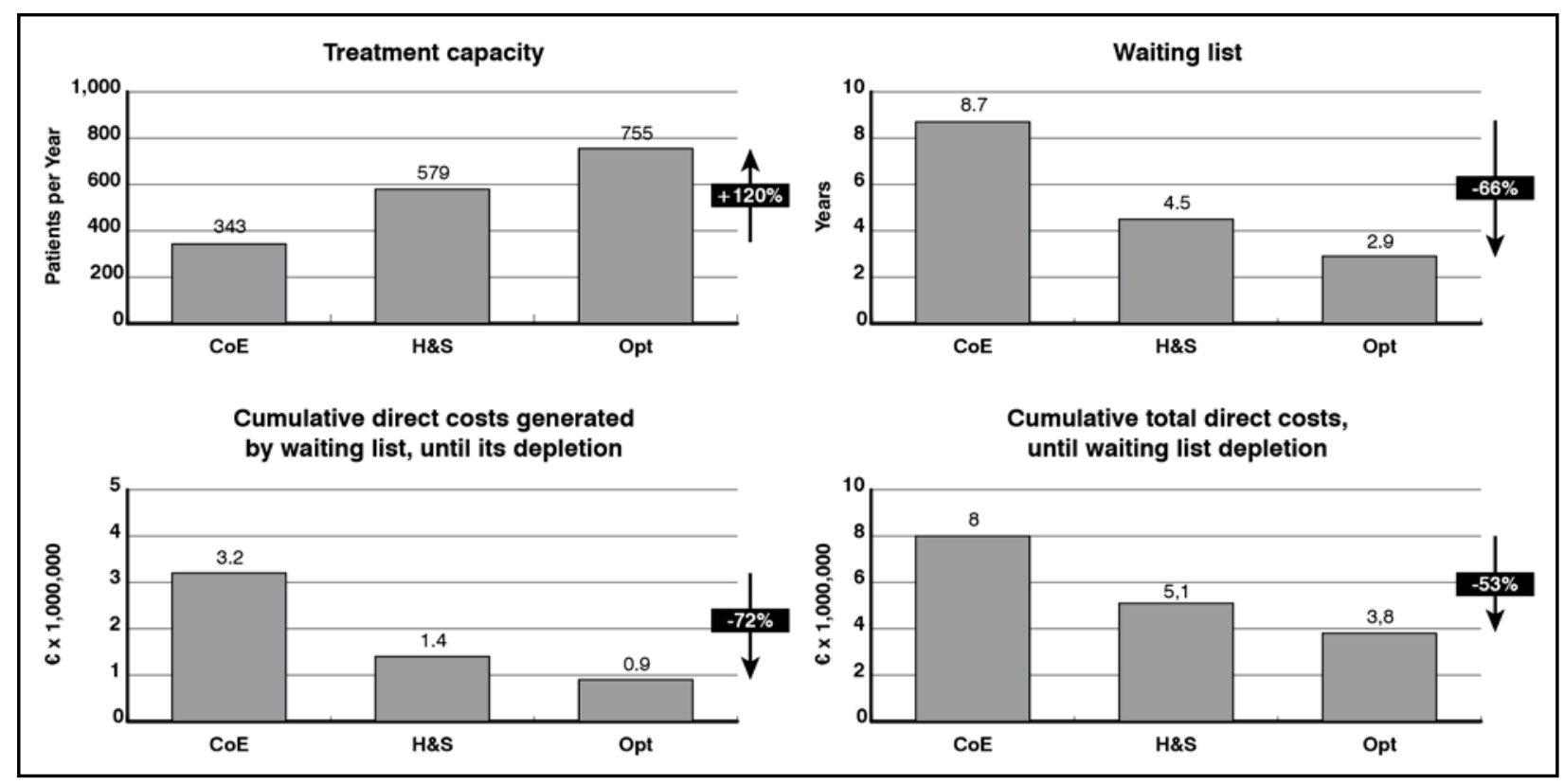

Figure 5. Parametric model results with new AIFA criteria $\mathrm{CoE}=$ Center of Excellence; H\&S = Hub\&Spoke; Opt = optimized

\begin{tabular}{lcc|ccc}
\hline & \multicolumn{2}{c}{ AIFA criteria $\mathbf{2 0 1 6}$} & \multicolumn{3}{c}{ AlFA criteria 2017 } \\
\cline { 2 - 6 } & CoE & H\&S & CoE & H\&S & Optimized \\
\hline Direct costs $(€)$ & 495,609 & 843,735 & 495,609 & 843,735 & 789,734 \\
• Diagnosis & 131,058 & 210,681 & 131,058 & 210,681 & 197,424 \\
- Treatment & 225,006 & 399,023 & 225,006 & 399,023 & 383,720 \\
• Follow-up & 139,545 & 234,031 & 139,545 & 234,031 & 208,590 \\
Waiting lists costs $(€)$ & 247,631 & 164,118 & 680,958 & 596,204 & 583,627 \\
Standby costs $(€)$ & 433,679 & 432,087 & - & - & - \\
\hline
\end{tabular}

Table IV. Comparison of costs data between the three models in the two alternative scenarios

timized" model costs are equal to $€ 4.6 \mathrm{M}$ over three years, showing an additional saving of $€ 0.7 \mathrm{M}(-13 \%)$ versus Hub\&Spoke (Figure 4).

A comprehensive summary of parametric model results, considering new AIFA access criteria, can be found in Figure 5 and Table IV. 


\section{DISCUSSION AND CONCLUSION}

This study represented a first, comprehensive mapping of the HCV patient pathway in the Italian healthcare system. As such, it allows to specifically understand effort and cost drivers for all resources involved, as well as to work as a reference, baseline or benchmark for future studies in the field.

Key evidence from cost driver analysis is related to differences between F3, F4 compensated and F4 decompensated. An average F3 patient would in fact cost about 30\% less than an average F4 decompensated one. Costs for F1 and F2 patients from the study are not representative of the general population (exceptional clinical record: liver transplant or severe extrahepatic diseases), but it can be reasonably expected they would be even lower than average F3 patient costs.

Untreated patients (deferred, stand-by) incurred a significant share (41\%) of direct costs; $72 \%$ of such share was related to stand-by patients, mostly represented by F0-F1-F2 patients periodically performing visits and lab tests to monitor their disease progression. The economic impact related to the management of those patients confirms the importance of eliminating $\mathrm{HCV}$ in the early fibrosis stages, even on an economic standpoint alone, thus providing a further rationale for AIFA's new access criteria.

The study also investigated the impact that different healthcare organizational models can have on HCV treatment, and particularly in accelerating elimination timing. We demonstrated how the Hub\&Spoke model deployed by PG-23 was able to increase the number of patients treated with DAAs over twelve months $(+69 \%)$ and to consequently reduce the waiting list $(-34 \%)$ and related costs $(-34 \%)$, compared to the CoE model required by Lombardy's Healthcare System. We also demonstrated that the Hub\&Spoke model can lead to successful HCV elimination (excluding currently undiagnosed patients) in Bergamo area within 4.5 year, compared to the 8.7 years of the CoE model. Finally, we identified three optimization opportunities to improve the Hub\&Spoke model; such opportunities would further reduce the waiting list to 2.9 years and allow additional cost savings, equal to $13 \%$ of direct costs.

Frequently, geographical differences among Italian regions led to different models and pathways to manage disease such as Hepatitis $\mathrm{C}$, also limiting the effective introduction of innovative treatments [13]. Some of the optimization opportunities used to design PG-23's "optimized" model can be potentially applied to other healthcare organizations at national and international level, regardless of them currently operating with a CoE, Hub\&Spoke or a different organizational model. Notably, the reduction of in-treatment monitoring visits and the streamlining of diagnosis visits do not require a specific organizational model to be in place. The study helps healthcare managers going beyond regional differentiation, showing clear evidence of potential benefits of implementing such optimization opportunities in their own context, to facilitate decision-making and a wider diffusion of best practices.

Limitations to the study are mainly related to population. In line with AIFA access criteria in place until March $30^{\text {th }}, 2017$, study population mostly included F3-F4 patients; a limited number of F1-F2 patients (7\% of study population) were treated with DAAs due to their exceptional clinical record (e.g. severe extrahepatic diseases). New access criteria are potentially going to change the composition of treated population, in favor of patients with lower fibrosis scores. This trend points towards the implementation of different care models to better match the needs of a wider, but less severe patient population. Care models may foresee further streamlining in patient pathways, for example through the elimination of mandatory Fibroscan tests, as DAAs are now indicated for any form of compensated liver disease regardless of fibrosis level [14], or also through an extended support role of GPs, in the diagnosis and treatment phases.

The study finally identified some areas for further investigation:

1. Launch of prevention initiatives to engage and raise disease awareness among high-risk patient groups;

2. Design and development of new access point network to focus on undiagnosed patients and facilitate early treatment. 


\section{Funding}

This study was funded by Gilead Science Srl. The views expressed here are those of the authors and not necessarily those of the funders.

\section{Conflicts of interest}

AA, CB, PC and CG are employees of Tefen \& Partners Consulting which has received financial support by Gilead Italy for the conduct of the study.

SF, LP, FM, RS, PDP, RB, MDM, and CN declare that they have no conflicts of interest in this research.

\section{REFERENCES}

1. World Health Organization. Hepatitis C. Fact sheet. Updated April 2017. Available at http:// www.who.int/mediacentre/factsheets/fs164/en/ (last accessed August 2017)

2. World Health Organization. Towards access 2030 - Medicine and Health Products program strategic Framework 2016-2030. Available at http://www.who.int/medicines/publications/ towards_access2030/en/ (last accessed August 2017)

3. ISTAT. Le principali cause di morte in Italia. Anno 2012. Available at: https://www.istat.it/ it/files/2014/12/Principali_cause_morte_2012.pdf (last accessed July 2017)

4. Wilkins T, Malcolm JK, Raina D, et al. Hepatitis C: diagnosis and treatment. Am Fam Physician 2010; 81: 1351-7

5. Poordad F, McCone J Jr, Bacon BR, et al.; SPRINT-2 Investigators. Boceprevir for untreated chronic HCV genotype 1 infection. N Engl J Med 2011; 364: 1195-206; http://dx.doi. org/10.1056/NEJMoa1010494

6. Lange CM, Jacobson IM, Rice CM, et al. Emerging therapies for the treatment of hepatitis C. EMBO Mol Med 2014; 6: 4-15; http://dx.doi.org/10.1002/emmm.201303131

7. Agenzia Italiana Del Farmaco. Bacheca atti amministrativi regionali. Available at http:// www.aifa.gov.it/content/bacheca-atti-amministrativi-regionali (last accessed August 2017)

8. Agenzia Italiana Del Farmaco. Nuovo algoritmo per la terapia dell'epatite C cronica. Available at http://www.agenziafarmaco.gov.it/content/1\%E2\%80\%99aifa-pubblica-il-nuovoalgoritmo-la-terapia-dell\%E2\%80\%99epatite-c-cronica (last accessed December 2017)

9. Regione Lombardia. Approvazione del documento di indirizzo per la gestione del nuovi farmaci antivirali per la terapia dell'epatite C cronica e per l'attivazione della rete regionale HCV. Decreto n. 7826 del 28/09/2015

10. Agenzia Italiana Del Farmaco. Ridefinizione dei criteri di trattamento per la terapia dell'Epatite C cronica. Determina n. 500/2017 del 24/03/2017. GU n.75 del 30-3-2017

11. Kondili LA, Romano F, Rolli FR, et al.; PITER Collaborating Group. Modeling CostEffectiveness and Health Gains of a "Universal" Versus "Prioritized" Hepatitis C Virus Treatment Policy in a Real-Life Cohort. Hepatology 2017; 66: 1814-25; http://dx.doi. org/10.1002/hep.29399

12. Agenzia Italiana Del Farmaco. Aggiornamento epatite C. Available at http://www.agenziafarmaco.gov.it/content/aggiornamento-epatite-c (last accessed August 2017)

13. Lanati EP, Lidonnici D, Gasbarrini A, et al. Cohort of HCV patients in Italy: sizing and treatments in a sample of Italian. ISPOR 17th Annual European Congress. Amsterdam: 2014

14. Rizzetto M. Un modello esportabile per l'eliminazione di HCV in Italia. ReAd files 2018; 19: 46-9 